\title{
News zum Mutterschutzgesetz
}

\begin{abstract}
Nach über 60 Jahren wird das Mutterschutzrecht aktualisiert, damit es besser in unsere heutige Arbeitswelt passt. Die Änderungen für eine Zahnarztpraxis halten sich jedoch im Rahmen.
\end{abstract}

Dr. Judith Schimann // Zahnärztin und freie Autorin, Kleinmachnow

$\mathrm{D}$ as Mutterschutzgesetz (MuSchG) für werdende und stillende Mütter gilt im Wesentlichen seit 1952, um das Leben und die Gesundheit von Mutter und Kind zu schützen. Um das Gesetz den jetzigen Gegebenheiten der Arbeitswelt anzupassen, wurde nun eine Aktualisierung des Mutterschutzrechts beschlossen. Am 30.03.2017 hat der Bundestag den Gesetzentwurf verabschiedet, in Kraft treten soll er in großen Teilen zum 01.01.2018. Doch zwei Regelungen sollen bereits nach Verkündigung des Gesetzes gelten:

1. Die Regelungen zur verlängerten Schutzfrist von zwölf Wochen nach der Geburt eines Kindes gelten nicht mehr nur bei Früh- und Mehrlingsgeburten, sondern auch bei Behinderungen des Neugeborenen.

2. Das viermonatige Kündigungsverbot nach der Geburt gilt jetzt auch für Mütter, die nach der zwölften Schwangerschaftswoche eine Fehlgeburt erleiden.

Das neue MuSchG bringt vor allem eine Ausweitung des geschützten Personenkreises mit. Es bezieht Frauen in betrieblicher Berufsbildung und Praktikantinnen sowie Schülerinnen und Studentinnen unter bestimmten Voraussetzungen mit ein, zudem noch weitere Arbeitnehmerkonstellationen.

Mehr Mitspracherecht soll es beim Verbot von Nacht- und Sonntagsarbeit geben. Auf Antrag kann eine Beschäftigung zwischen 20:00 und 22:00 Uhr sowie nachts und feiertags mit Einwilligung der Schwangeren möglich sein.

Bei der Entgeltabrechnung ändert sich nichts. Es gibt weiterhin eine Zuschusspflicht zum Mutterschaftsgeld in den Schutzfristen sowohl vor als auch nach der Geburt. Auch das konkrete Vorgehen bei der Mitteilung über eine Schwangerschaft bleibt für den Arbeitgeber ohne Veränderung. Allerdings werden die Regelungen der bisherigen Verordnung zum Schutze der Mütter am

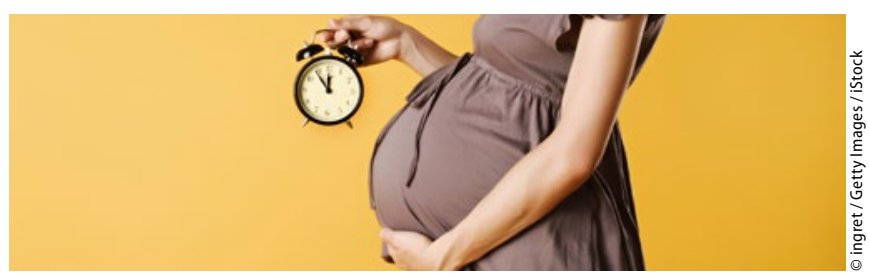

Arbeitsplatz (MuSchArbV) in das MuSchG integriert - und damit auch sämtliche Beschäftigungsverbotsgründe, insbesondere die Gefährdung durch Umgang mit Gefahrstoffen. Dazu wurden die gesundheitlichen Vorschriften auf der Grundlage aktueller arbeitsmedizinischer Erkenntnisse überarbeitet und eine für Mutter und Kind auszuschließende sogenannte unverantwortbare Gefährdung eingeführt. Im Gesetz gibt es für die verschiedenen Gefährdungen konkrete Beispiele, aus denen deutlich wird, was in einem speziellen Fall unter einer unverantwortbaren Gefährdung zu verstehen ist. Für schwangere und stillende ZFA ist also auch nach dem neuen Mutterschutzgesetz wegen des Umgangs mit Gefahrstoffen und der Infektionsgefahr eine Tätigkeit am Stuhl auszuschließen. Auch die Beschäftigung einer schwangeren angestellten Zahnärztin wird weiterhin nicht möglich sein. Dagegen werden selbständige Zahnärztinnen auch zukünftig nicht vom Mutterschutzgesetz erfasst. Ihre Berufstätigkeit bleibt ohne Einschränkung möglich.

Allerdings wird mit der Neuregelung der Arbeitgeber mehr denn je in die Verantwortung genommen, Beschäftigungsverbote zu reduzieren. Er soll geeignete Maßnahmen ergreifen, um die Weiterbeschäftigung zu ermöglichen, zum Beispiel durch Umsetzung der Mitarbeiterin in den administrativen Teil der Zahnarztpraxis. Für angestellte Zahnärztinnen sind andere Aufgaben nur zumutbar, wenn sie ihrer Qualifikation entsprechen. Erst wenn dies in der Praxis nicht möglich ist, ist gegebenenfalls ein Beschäftigungsverbot auszusprechen. Eine Kündigung ist während der Schwangerschaft nicht möglich. Der Kündigungsschutz besteht auch nach der Geburt bis zum Ende der Schutzfrist und mindestens vier Monate.

\section{Hier steht eine Anzeige.}

